\title{
Characteristics of condensed gas moderators for the generation of very slow polarized muons
}

\author{
E. Morenzoni \\ Paul Scherrer Institut, CH-5232 Villigen PSI, Switzerland
}

Th. Prokscha

Paul Scherrer Institut, CH-5232 Villigen PSI, Switzerland and Physik Institut, Universität Heidelberg, D-69120, Germany

A. Hofer

Paul Scherrer Institut, CH-5232 Villigen PSI, Switzerland and Fakultät für Physik, Universität Konstanz, D-78434 Konstanz, Germany

B. Matthias ${ }^{\mathrm{a})}$

Physik Institut, Universität Heidelberg, D-69120 Heidelberg, Germany

M. Meyberg, ${ }^{\text {b) }}$ Th. Wutzke, ${ }^{\mathrm{c})}$ and H. Glückler

Paul Scherrer Institut, CH-5232 Villigen PSI, Switzerland

M. Birke

Paul Scherrer Institut, CH-5232 Villigen PSI, Switzerland and Institut für Metallphysik und Nukleare

Festkörperphysik, Technische Universität Braunschweig, D-38106 Braunschweig, Germany

J. Litterst

Institut für Metallphysik und Nucleare Festkörperphysik, Technische Universität Braunschweig,

D-38106 Braunschweig, Germany

Ch. Neidermayer and G. Schatz

Fakultat für Physik, Universität Konstanz, D-78434 Konstanz, Germany

(Received 18 November 1996; accepted for publication 3 January 1997)

Motivated by the possibility of using condensed gas moderators to produce very slow (epithermal) polarized muons we have studied the moderator properties of rare gas solids and solid $\mathrm{N}_{2}$ thin films as a function of the growing parameters (deposition temperature and rate, heat treatment) and of the film thickness. For Ar and $\mathrm{Kr}$ moderators the moderation efficiency is found to depend on the growth temperature and an annealing effect is observed. The dependence can be interpreted in terms of the changes in granularity and porosity of the condensed gas layers when the growth parameters are varied. From the thickness dependence of the moderation efficiency the escape depth of the very slow muons was determined. Its large value indicates a suppression of electronic energy loss mechanisms for the epithermal muons. The time dependent decay properties of the moderators were also investigated and found to depend solely on the rest gas pressure. At a pressure of $10^{-10} \mathrm{mbar}$ the moderation efficiency remains stable over a period of days. (C) 1997 American Institute of Physics. [S0021-8979(97)00308-3]

\section{INTRODUCTION}

The moderation of energetic polarized positive muons $\left(\mu^{+}\right)$in a film of a solid van der Waals gas and the escape from its surface presently offers the most efficient mechanism to generate polarized muons of a few eV kinetic energy in vacuum (epithermal $\left.\mu^{+}\right){ }^{1,2}$ With a so-called surface muon beam $(\sim 4 \mathrm{MeV})$ implanted in a planar moderator, efficiencies to convert fast muons into slow ones of the order of $10^{-4}-10^{-5}$ have been obtained. This is about two orders of magnitude larger than efficiencies obtained from single ionic crystal insulators or from metals.

Making use of a moderator as a very slow $\mu^{+}$source, beams of polarized muons with tunable energy between a few $\mathrm{eV}$ and a few tens of $\mathrm{keV}$ are being developed. ${ }^{3}$ The

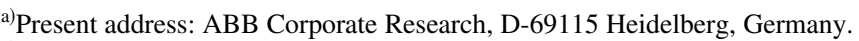
b) Present address: DMP AG, CH-8604 Volketswil, Switzerland.

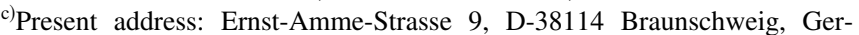
many.
}

availability of such beams permits the field of application of the various muon spin rotation $(\mu \mathrm{SR})$ techniques to be extended to thin-film and surface physics studies. These techniques make use of $\mu^{+}$as microscopic magnetic probes by detecting the positrons originating from their anisotropic decay. ${ }^{4}$ They are presently based on the implantation of energetic polarized $\mu^{+}$and limited to bulk studies. By tuning the energy in the $\mathrm{eV}$ to $\mathrm{keV}$ energy range the implantation depth can be varied between a fraction of a $\mathrm{nm}$ and several hundreds of $\mathrm{nm}$, thus adding depth resolution to the $\mu \mathrm{SR}$ method.

The physics of $\mu^{+}$moderation and the dynamics of epithermal muons in condensed gas solids is not well understood. Analogous to the case of $e^{+}$moderation $^{5,6}$ the relatively large moderation efficiency is attributed to the sizeable reduction of the muon energy loss rate (or stopping power) once the muons have reached energies in the $\mathrm{keV}$ to $\mathrm{eV}$ region. The reduction is explained by the presence of a minimum threshold energy for electronic energy loss producing 


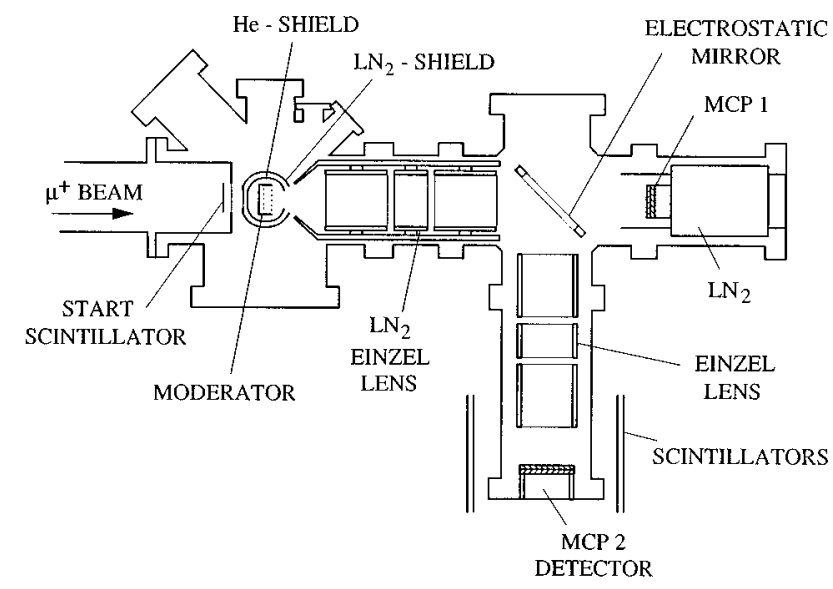

FIG. 1. Schematic view of the UHV apparatus with cryostat used for the moderation studies and the very slow $\mu^{+}$production.

mechanisms (ionization, excitation, electron pickup and loss by $\left.\mu^{+}\right)$. However, to date no experimental evidence of this mechanism for heavy projectiles such as muons (muon mass $m_{\mu} \cong 207 m_{e}$ ) in condensed gas solids exists. Since the slowing down process is very fast (typically a few ps), no depolarization occurs during this stage, which is an essential point for many applications. ${ }^{2}$

The moderation technique is routinely employed in the production of very slow $e^{+}$beams. As opposed to the case of positron moderation, where alternative noncryogenic moderators exist, the only practicable possibility to produce very slow muons, at the present, seems to be the use of cryocrystals. Therefore, besides improving the understanding of the moderation mechanisms, it is important to assess the characteristics of condensed gas moderators and to find the optimum operating conditions. Both aspects open the possibility of producing more efficient moderators.

In this paper we report on investigations of the dependence of the moderation efficiency on parameters such as thickness, deposition rate, temperature and heat treatment of solid $\mathrm{Ar}, \mathrm{Kr}, \mathrm{Xe}$, and $\mathrm{N}_{2}$ films. The thickness dependence measurements allow the determination of the escape depth of the epithermal $\mu^{+}$and yield insight into the mechanisms by which the $\mu^{+}$are slowed down and emitted from the solidified gas. The results as a function of the growth conditions are discussed by considering the structure of the film and its influence on the moderating mechanisms. A possible critical point of cryogenic moderators is their long-term stability since, due to the low sublimation energy and the large adsorption capacity of vacuum contaminants, a degradation of the moderation efficiency with time may be expected. This question has been addressed by studying the decay properties of the moderation efficiency under different vacuum and experimental conditions.

\section{EXPERIMENTAL DETAILS}

The apparatus for this experiment is shown schematically in Fig. 1. A secondary beam line of the Paul Scherrer Institute (PSI) accelerator complex is tuned to deliver muons with a momentum of $27.7 \mathrm{MeV} / \mathrm{c}$, corresponding to a kinetic energy of $\sim 3.6 \mathrm{MeV}$, and a momentum width $\Delta p / p$ between $1 \%$ and $3 \%$. After collimation, the incident $\mu^{+}$ traverse a thin $(200 \mu \mathrm{m})$ plastic scintillator producing the start signal of a time-of-flight (TOF) measurement. They enter an ultra-high vacuum (UHV) chamber through a $50 \mu \mathrm{m}$ stainless-steel window, which separates the UHV of the slow $\mu^{+}$apparatus from the poorer vacuum $\left(\sim 10^{-5}\right.$ mbar $)$ of the beam line.

The central component is the cryogenic moderator. It consists of a thin Al substrate (thickness $250 \mu \mathrm{m}$, purity $99.999 \%$ ) cooled by a He flow cryostat and covered on the downstream side with a few $100 \mathrm{~nm}$ thick layer of a condensed van der Waals gas. Two shrouds of gold plated oxygen-free high-conductivity (OFHC) copper [the outer one cooled by a liquid nitrogen $\left(\mathrm{LN}_{2}\right)$ reservoir and the inner one by the liquid helium reservoir] surround the moderator to shield the film from the thermal radiation of room temperature surfaces. Only an opening downstream of the moderator is left in the shielding for the extraction of slow $\mu^{+}$. To prevent the moderator from being exposed to too much thermal radiation, the elements facing the opening (electrostatic lens and beam dump of the nonmoderated $\mu^{+}$) are cooled to $\mathrm{LN}_{2}$ temperature.

In the substrate, about $50 \%$ of the beam is stopped. A small but not negligible fraction of energetic $\mu^{+}$is slowed down to epithermal energies in the condensed gas layer and is subsequently emitted. The cold metal substrate is electrically insulated with a sapphire disk and can be raised to voltages as high as $20 \mathrm{kV}$. This allows one to accelerate and extract the very slow $\mu^{+}$from the production region. These particles are deflected by $90^{\circ}$ with respect to the incoming beam by an electrostatic mirror and are focussed by two electrostatic "einzel" lenses onto a microchannel plate detector (MCP 2). MCP 2 gives the stop signal of the TOF measurement. The muons can be unambiguously identified using the signal of MCP 2 in coincidence with the delayed signal from one of the four pairs of scintillator paddles which detect the positron originating from the $\mu^{+}$decay (lifetime $2.2 \mu \mathrm{s})$. The epithermal muons appear as a pronounced peak in the TOF spectrum. The area of the peak, normalized to the number of incoming surface muons and corrected for transport and detection efficiencies and electronics deadtime, gives the moderation efficiency of an energetic muon beam with defined momentum and momentum bite. For the use of very slow $\mu^{+}$as magnetic probes, a holder for various types of thin film samples can be mounted in place of the MCP.

Base pressures of about $10^{-9}$ mbar were obtained without coolants in the apparatus. With a cold cryostat, the pressure was as low as $7 \times 10^{-11}$ mbar (pressure gauge reading). Some measurements were also performed in the $10^{-8}$ $10^{-9}$ mbar range to study the decay characteristics of the moderator efficiency as a function of pressure.

The thin film layer is formed by condensing research purity gas on the cold $\mathrm{Al}$ substrate. The gas, regulated with a high precision leak valve, is admitted into the vacuum chamber through a directional gas-doser mounted on a manipulator which, for the deposition procedure, is positioned in front of the substrate at a distance of $\sim 10 \mathrm{~cm}$. Within a few minutes of ending the film growth procedure, the residual pres- 
sure generally returns to the $10^{-10}$ mbar range. The gas composition in the UHV chamber is monitored with a quadrupole mass spectrometer.

The thickness of the film is measured with a quartz microbalance (standard frequency shift method) and additionally, in some cases, with an interferometric method. The quartz crystal is placed adjacent to the substrate and is also cooled by the cryostat. By letting gas into the UHV chamber, a film also condenses onto the crystal. The thickness of the film accumulated on the quartz is directly proportional to the film thickness on the Al substrate, where the proportionality constant has to be determined by a series of calibration measurements by mounting the quartz crystal at the substrate position. Below $\sim 20 \mathrm{~K}$ the frequency of the crystal is practically temperature independent. The temperature of the moderator substrate is measured with Lake Shore Si-diodes specified to have $\pm 0.25 \mathrm{~K}$ absolute accuracy in the relevant temperature range and is stabilized to better than $0.1 \mathrm{~K}$. The thickness of the condensed gas layer is found to be stable to within $\sim 0.1 \mathrm{~nm}$. With the interferometric method the growth of the film during deposition can be monitored. ${ }^{7}$ Light from a $\mathrm{He}-\mathrm{Ne}$ laser $(632.8 \mathrm{~nm})$ is either reflected at the $\mathrm{Al}$ surface or at the surface of the condensing gas layer. During film deposition, the reflected intensity, measured with a photodiode, shows a typical interference pattern structure from which the film thickness can be deduced. The two methods give consistent results within 15\%. Furthermore, depending on gas and deposition parameters, the thickness experimentally determined corresponds to between $70 \%$ and $95 \%$ of the value calculated from the amount of gas impinging on the substrate during deposition and assuming a sticking probability of unity. For the analysis we used the data obtained with the quartz microbalance, which is more sensitive in the investigated thickness range. The time-of-flight and other relevant detector information are recorded event by event, whereas the slowly varying parameters such as temperature, pressure, rest gas composition, and thickness are monitored and written to a file every few minutes.

\section{RESULTS AND DISCUSSION}

\section{A. Thickness dependence}

Figure 2 shows the yield of slow $\mu^{+}$emitted from $\mathrm{Ar}$ and $\mathrm{Kr}$ moderators (defined here as the number of very slow $\mu^{+}$detected by MCP 2 per $10^{8}$ incoming fast $\mu^{+}$) as a function of the layer thickness. The absolute moderator efficiency is obtained in this case by multiplying the numbers by $3 \times 10^{-8}$. The Ar moderator was grown at $21 \mathrm{~K}$ at a partial pressure of $4 \times 10^{-5}$ mbar and the $\mathrm{Kr}$ one at a temperature of $26 \mathrm{~K}$ and a pressure of $1 \times 10^{-5}$ mbar. In both cases, the yield first increases with increasing thickness until it saturates at values of a few hundred $\mathrm{nm}$. This dependence can be understood in terms of a very simple model which distinguishes two stages of the moderation and emission processes. In a first step, as a result of the statistical nature of the slowing down process, some $\mu^{+}$become epithermal inside the frozen gas layer. Their density $n$ (number of epithermal $\mu^{+}$per projectile per unit thickness) is constant, since the width of the $\mu^{+}$stopping distribution $(\sim 20 \mu \mathrm{m})$ is much
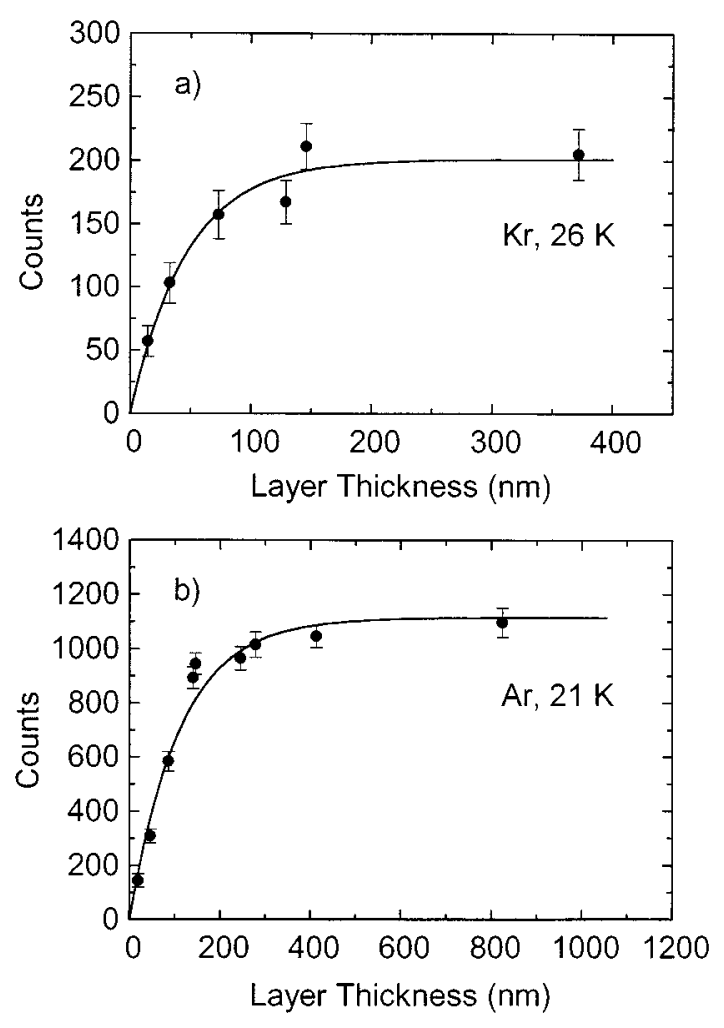

FIG. 2. Dependence of the epithermal muon yield on the thickness of the condensed gas. (a) solid $\mathrm{Kr}$, (b) solid Ar. The solid lines represent a fit assuming a uniform generation density and an exponential attenuation law.

larger than the typical condensed gas film thickness. From a computer simulation we obtain for Ar $n \cong 2 \times 10^{-5}$ and for $\mathrm{Kr} n \cong 2.3 \times 10^{-5}$ slow $\mu^{+} /$projectile/nm. In a second step, the $\mu^{+}$may move towards the surface and are emitted with a certain probability. Thus, in a simplified one-dimensional approach and without considering energy and angular dependence, the epithermal $\mu^{+}$yield $N_{\mu}$ as a function of the condensed gas thickness $d$ may be written as:

$$
N_{\mu}(d)=n \int_{0}^{d} P(x) d x,
$$

where $P(x)$ represents the probability for a $\mu^{+}$produced at a depth $x$ from the surface to move toward and escape from the surface. Assuming an exponential attenuation law, the escape probability is given by $P(x)=P(0) \exp ^{-x / L}$. Here $P(0)$ takes into account all possible epithermal muon losses such as $\mu^{+}$neutralization inside the layer or at the surface and $L$ is the average escape depth for very slow muons. By inserting $P(x)$ in eq. (1) one obtains:

$$
N_{\mu}=P(0) \operatorname{Ln}\left(1-\exp ^{-d / L}\right),
$$

which has been used to fit the data in Fig. 2. We find for $\operatorname{Ar} L=(111 \pm 8) \mathrm{nm}$ and for $\operatorname{Kr} L=(47 \pm 9) \mathrm{nm}$.

These large escape depths and the related large moderation efficiencies are a direct manifestation of the stopping power (energy loss per unit path) for a charged particle in a perfect insulator such as solid Ar or solid Kr. Up to date, even for readily available particles such as protons, experimental data are scarce at very low energies. Also, as far as 


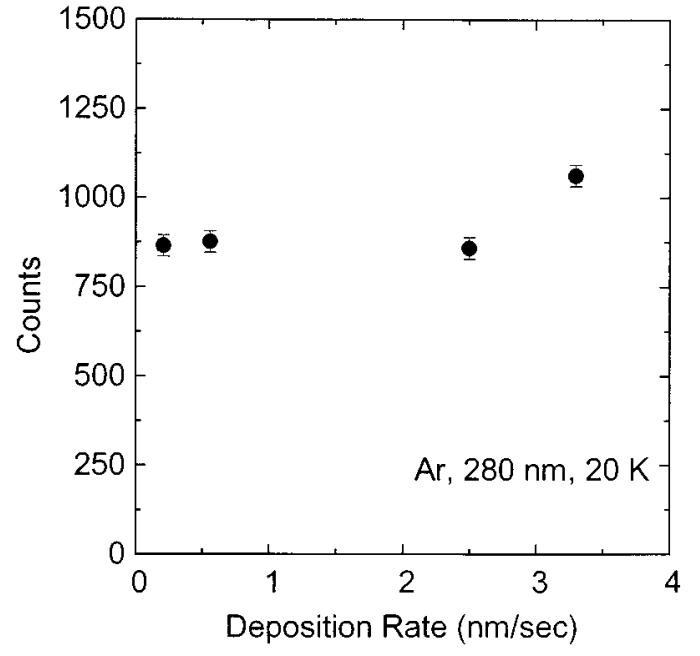

FIG. 3. Deposition rate dependence of the epithermal muon yield from an Ar moderator.

theory is concerned, for insulators, no easily tractable procedures to calculate the stopping power are available. Two mechanisms contribute to the energy loss of a heavy particle traversing matter. The first one is given by the interaction of the projectile with electrons in the solid, resulting in electronic excitation processes of the projectile and target atoms (electronic stopping power). ${ }^{8}$ At very low velocities, a second important contribution to the slowing down process arises from the energy transfer to atoms as a whole via elastic collisions (nuclear stopping power). Supported by several model calculations, the standard treatment of energy loss in matter assumes that the first contribution is proportional to velocity down to very low energies. According to these models, even for $10 \mathrm{eV} \mu^{+}$the electronic stopping power is dominant; ${ }^{8}$ however, the linear velocity dependence implies that the minimum energy transferred to the electrons is much smaller than the projectile energy. This is evidently not the case in wide-band-gap insulators such as the van der Waals solids. The large escape depth value clearly demonstrates that the stopping power of heavy particles in insulators, in the low velocity regime, sizably departs from the linear velocity dependence. The departure can be roughly quantified by considering that $L \leqslant \int_{E_{\mathrm{in}}}^{E_{\mathrm{fin}}}(d E / d x)^{-1} d E$. Using the well known computer code "Transport of Ions in Matter", $(\mathrm{TRIM})^{8}$ to calculate the muon stopping power $(d E / d x)$ and choosing $E_{\mathrm{in}} \simeq 10 \mathrm{eV}$ and $E_{\mathrm{fin}} \simeq 1 \mathrm{eV}$ one would expect, for Ar as well as for $\mathrm{Kr}$, an upper limit for $L$ of $\sim 3 \mathrm{~nm}$. The measured escape depths are significantly higher than this value. On the other hand, reasonable agreement is obtained if, in a very crude approximation, only the energy loss due to elastic collisions is taken into account. It therefore appears that the electronic stopping power of $\mu^{+}$in solid $\mathrm{Ar}$ and $\mathrm{Kr}$ is significantly less than predicted and that the final stage of moderation is completely dominated by elastic collisions. More refined comparisons are presently not possible, due to the lack of theoretical models or estimates analyzing the interaction of the moving $\mu^{+}$with the atoms of the van der Waals solid.
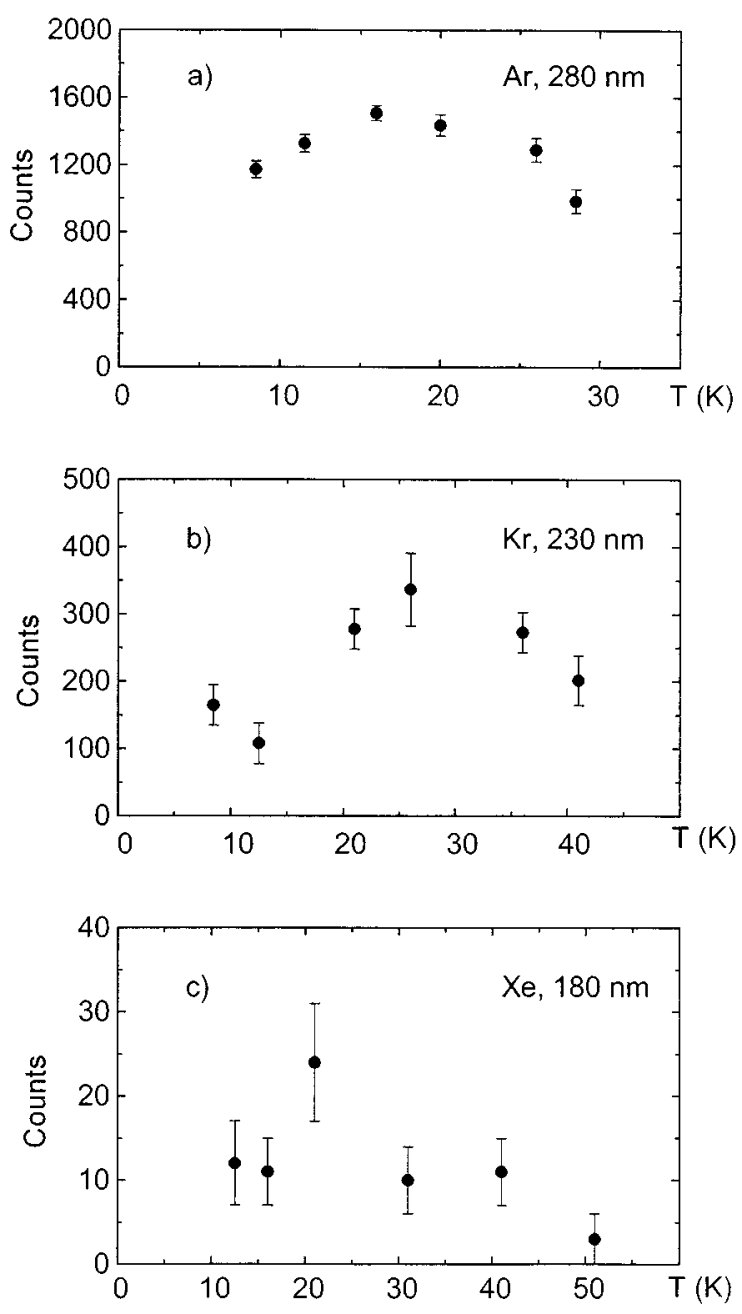

FIG. 4. Measurement of the very slow $\mu^{+}$yield vs deposition temperature. (a) Ar, (b) Kr, (c) Xe.

\section{B. Rate and temperature dependence}

For the following data presentation and discussion we define a deposition temperature $T_{d}$ as the substrate temperature during deposition and a moderator temperature $T_{m}$ as the temperature at which the measurements are performed. We first investigated the dependence of the slow $\mu^{+}$yield on the deposition rate. Figure 3 shows the result for a $280 \mathrm{~nm}$ thick Ar layer at $T_{d}=T_{m}=20 \mathrm{~K}$. Within the rate variation of more than an order of magnitude, corresponding to effective Ar vapor pressures between $3.5 \times 10^{-6}$ and $7 \times 10^{-5}$ mbar, the data show no pronounced dependence with a possible slight preference for large deposition rates. Therefore, most of the measurements presented in this work were taken with films grown at a partial gas pressure in the $10^{-5}$ mbar range over a few minutes.

In Fig. 4 the dependence on deposition temperature for $\mathrm{Ar}, \mathrm{Kr}$, and $\mathrm{Xe}$ moderators is plotted (in these cases $\left.T_{d}=T_{m}\right)$. For $\mathrm{Ar}$ and $\mathrm{Kr}$, increasing the temperature results first in moderators that are superior to those deposited at the minimum temperature, then further increasing the temperature gives smaller efficiencies. For Ar a temperature of $\sim 15 \mathrm{~K}$ and for $\mathrm{Kr}$ a temperature of $\sim 30 \mathrm{~K}$ turn out to be 

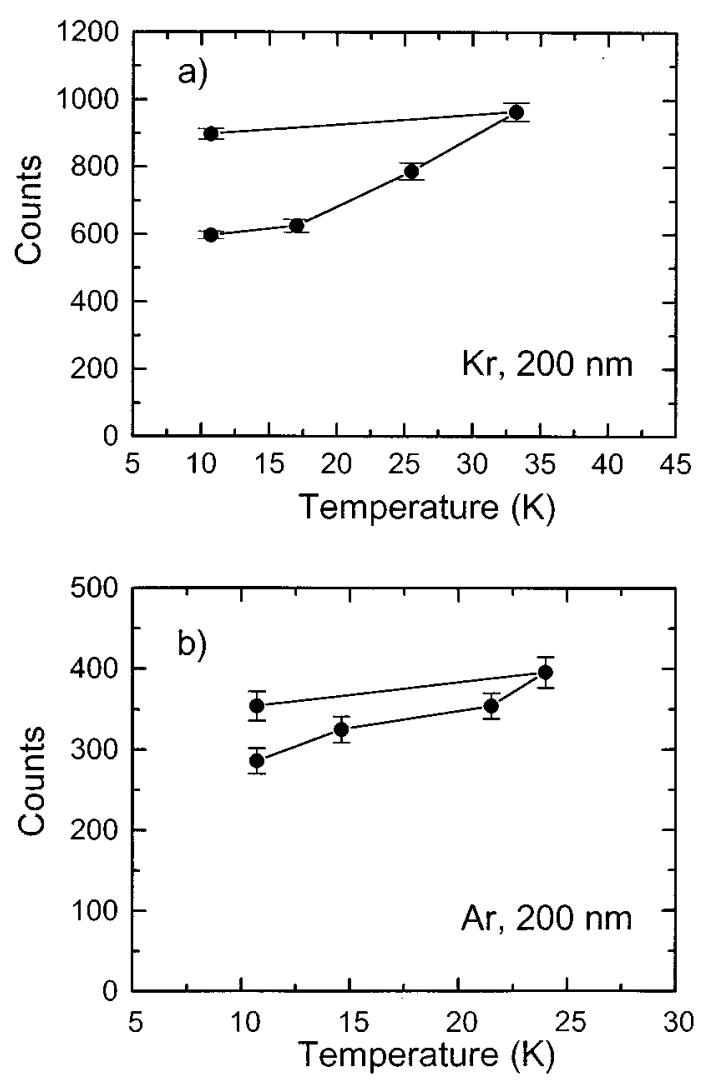

FIG. 5. Yields of epithermal muons during temperature cycling. The moderator was grown at the lowest temperature, then heated up slowly and finally recooled. (a) $\mathrm{Kr}$, (b) Ar. The solid line indicates the temporal sequence in which the data were taken.

optimal. For Xe no pronounced temperature dependence of the moderation efficiency is observed. It should be noted that, in all cases, at the highest investigated temperature noticeable sublimation sets in and the films become unstable as is also indicated by the partial pressure increase in the quadrupole mass spectrometer.

Since the very slow $\mu^{+}$emission in $\mathrm{Ar}$ and $\mathrm{Kr}$ was found to depend on $T_{d}$ we also investigated the effects of temperature cycling. The temperature range where these experiments can be performed without the crystals subliming in the UHV system is quite limited in the case of thin layers. Nevertheless, for Ar and $\mathrm{Kr}$, as shown in Fig. 5, an effect could be observed. A fresh Ar or Kr layer was deposited at low temperature. Then the moderator was slowly warmed up to below the sublimation temperature (we define the sublimation temperature as the temperature where the partial pressure reaches $\sim 10^{-5}$ mbar. At this pressure the thin condensed gas layer disappears within a few minutes). The warming up brings a continuous increase in yield, of $\sim 20 \%$ in the case of $\mathrm{Ar}$ and of $\sim 50 \%$ in that of $\mathrm{Kr}$, in agreement with the measured temperature dependence. By recooling the film to the lowest temperature the higher yield could be "frozen" and maintained, indicating that annealing produces structural changes in the film. The same effect was obtained by performing the annealing procedure within a few minutes. In contrast, $\mathrm{N}_{2}$ showed no improvement upon annealing between 10 and $20 \mathrm{~K}$.
The experimental findings can be put in relation to the morphological and structural characteristics of the thin films of condensed gases which are known to depend on the growth conditions. Due to their basic simplicity much experimental work has been carried out especially with rare gas solids (RGS). ${ }^{9,10}$ The main features can be summarized as follows. Thin films grown from the vapor have polycrystalline structure. As for the bulk, crystallization of RGS occurs in face-centered cubic lattices, but polycrystalline specimens contain more grain boundaries, defects and voids and are generally more porous than when grown from the liquid. Films deposited at higher temperature just below the sublimation temperature have larger grain sizes and are less defective than films produced on a cold substrate, but the grain size in the substrate plane is generally limited to a few times the film thickness. From the sharpness of electron diffraction patterns it has been observed that in an Ar film deposited at lower substrate temperature and annealed to $\sim 28 \mathrm{~K}$ some recrystallization occurs which does not disappear after cooling the substrate back down to $\sim 5 \mathrm{~K}^{11,12}$

If we compare these properties with our results we may conclude that larger moderation efficiencies in $\mathrm{Ar}$ and $\mathrm{Kr}$ are correlated to higher degrees of crystallization (larger grain size, smaller number of defects). It seems, therefore, that the quality improvement of the thin film, which is achieved by increasing the temperature, is directly reflected in an increased moderation efficiency. This is particularly evident from the annealing measurements. We can only speculate how grain size and defects influence the transport properties of epithermal $\mu^{+}$and their moderation. Since epithermal $\mu^{+}$are still very hot, it is very improbable that defects and boundaries act as effective trapping sites. Presumably grain boundaries and defects introduce, due to the large polarizability of rare gas atoms, local dipole fields generating long range electrostatic potentials and thus increase the elastic scattering cross section and the nuclear energy loss per collision. Thus within the bounds of this mechanism it is clear that a temperature increase would favor a moderation increase.

However, if grain size and defects were the only relevant parameters, we would expect a steady increase of the moderation efficiency with increasing $T_{d}$, in disagreement with the results for $\mathrm{Ar}$ and $\mathrm{Kr}$. Other physical properties that are temperature dependent are the density and the morphology, which affect the porosity and the adsorption properties of the deposited film. It is known, e.g., from Ref. 13, that the density of films of RGS and other van der Waals solids strongly depends on $T_{d}$. For $T_{d}$ above a value $T_{\mathrm{ch}}$, which is characteristic for each gas, the solid layers have the same density as the respective bulk crystals. For $\mathrm{Ar}, \mathrm{Kr}, \mathrm{Xe}$, and $\mathrm{N}_{2}$ $T_{\mathrm{ch}} \cong 18,29,40$ and $18 \mathrm{~K}$, respectively, corresponding to about $2 / 3$ of the sublimation temperature. Below $T_{\text {ch }}$, however, a decrease of the density with decreasing $T_{d}$, which can be as large as $30 \%$, is observed. If $T_{d}$ is further reduced, a distinct range of constant density appears in some cases (Ar, Kr, Xe). This corresponds fairly well to maximum adsorption capacity indicating an open-pore structure in this temperature range. ${ }^{14,15}$ The effect of open pores would consist primarily of enlarging the active surface area from which 
the epithermal $\mu^{+}$are emitted and hence of increasing the moderation efficiency. Also consistent with this interpretation is the slight preference for high deposition rates at a fixed temperature, since fast growing layers possess greater disorder and porosity. Reducing the density further results in a diminished adsorption capacity indicating a closing of the porous structure.

For the observed maximum of the efficiency versus deposition temperature we therefore propose as an explanation the interplay of two opposite effects, namely the competition between the efficiency increase due to the increase of grain size with temperature and the decrease due to the closing of the porous structure. It should be noted that, in first approximation, the change in density with temperature should itself not influence the moderation efficiency. This can be understood by considering that the slow $\mu^{+}$yield is proportional to the product of the stopped $\mu^{+}$density and escape depth [(as can be easily seen from equation (2) for $d \gg L)]$ and that the first quantity is proportional to the mass density whereas the latter is inversely proportional.

It is instructive to compare our results on $\mu^{+}$moderation in RGS with previous results on $e^{+}$moderation. While appropriate analogies can be drawn between $e^{+}$and $\mu^{+}$, their mass difference has important implications in the transport characteristics of both particles in condensed matter.

For a decade RGS have proven to be highly efficient $e^{+}$moderators. ${ }^{16}$ The question of the optimal growth conditions has been addressed only recently. ${ }^{17-19}$ Optimum efficiency has been reported for $\mathrm{Ar}$ at $\sim 16 \mathrm{~K}$ and $\mathrm{Kr}$ at $\sim 35 \mathrm{~K}$ in good agreement with the $\mu^{+}$results. Proper annealing has a more pronounced effect for $e^{+}$than for $\mu^{+}$. For instance, the $e^{+}$moderation efficiency of a $\mathrm{Kr}$ moderator deposited at $4.5 \mathrm{~K}$ improves by a factor 2.5 after warming to $43 \mathrm{~K}$ and cooling down again. These results suggest that the structural and morphological characteristics of the condensed layer have similar qualitative influences on both particles as far as the transport is concerned; however, quantitative differences exist. They can be explained by the different moderation dynamics which is evidenced by considering the de Broglie wavelength $\lambda$ of the particles in the relevant energy regime. Whereas even at $10 \mathrm{eV}$ the $\mu^{+}$behaves as a pointlike classical particle $\left(\lambda_{\mu}=0.03 \mathrm{~nm}\right)$ the size of the wave function of epithermal $e^{+}$exceeds the atomic radius. Hot $e^{+}$are delocalized and their quantum mechanical nature cannot be neglected. Therefore the granular structure and the presence of grain boundaries and defects have more pronounced effects on the $e^{+}$transport than on the $\mu^{+}$transport. This causes the similarity in the temperature and annealing dependence and explains that the effects are more pronounced for $e^{+}$. In this respect, another important difference is that epithermal $e^{+}$ lose energy to phonons, which are also likely to be sensitive to the crystallites quality, and not to individual particle collisions as is the case for muons.

\section{Decay properties}

The long term stability of the moderator and the decay of the efficiency represent an important practical aspect for the development of a very slow $\mu^{+}$beam based on condensed gas moderators. Additionally, the understanding of these

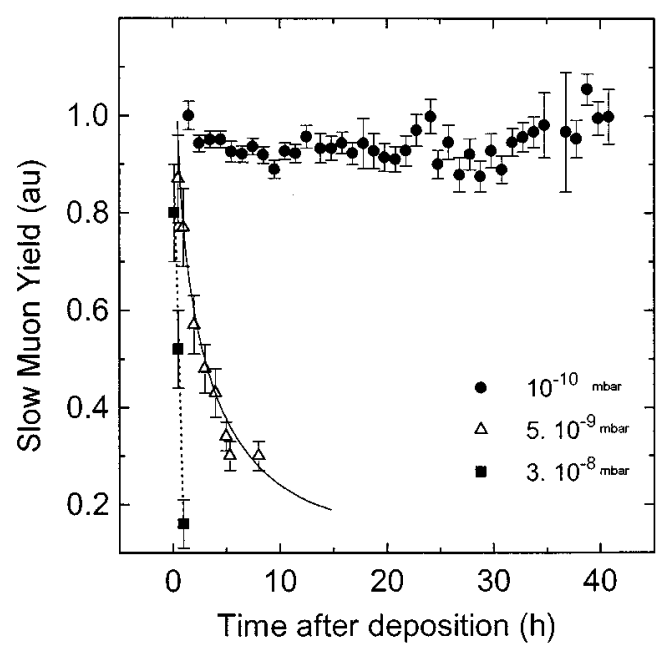

FIG. 6. Normalized yields of very slow $\mu^{+}$from solid Ar as a function of time after layer deposition for different chamber pressures.

properties can also give insight into the processes governing the generation of epithermal $\mu^{+}$. The solidified film is submitted to the bombardment of energetic $\mu^{+}$and to the action of the adsorption of contaminants from the residual gas molecules. Both processes may produce a decay in time of the moderation properties in one case by degrading or by eroding the layer and in the other case by attenuating the probability that a slow $\mu^{+}$created inside the moderator may be transmitted through the surface layer.

The influence of the residual gas is demonstrated in Fig. 6 which shows the moderation efficiency of $\mathrm{Ar}$ as a function of time after layer deposition for three different chamber pressures. At a pressure of $10^{-8}$ mbar (mainly due to water) the yield decays rapidly and within few hours no epithermal $\mu^{+}$are observed. A pressure in the $10^{-10}$ mbar range is necessary and sufficient to maintain stable operating conditions over several days. This is also the case for $\mathrm{N}_{2}$ moderators. The total pressure is a crucial but relatively rough parameter to characterize the complexity of possible mechanisms taking place in the adsorbate system formed by vacuum contaminants on the cryogenic surface. A detailed understanding of the specific role played by the different residual gas components (mainly hydrogen, water, $\mathrm{CO}$ and $\mathrm{CO}_{2}$ ) is beyond the scope of this work; nevertheless, some data have been obtained as a byproduct of our measurements. Neutrals and ions desorbed or sputtered by the projectiles stem from the first monolayers of the moderator. Whereas we cannot observe neutrals, the ion species can be identified by our electrostatic transport system with the TOF measurement which is sensitive to the mass to charge ratio.

Preliminary studies show, beside $\mathrm{H}^{+}$, the presence of strong desorbing clusters from the surface of all RGS moderators consisting of a proton and $n$ water molecules $\left[\left(\mathrm{H}_{2} \mathrm{O}\right)_{n} \mathrm{H}^{+}\right.$, protonated water]. No water ion clusters, $\mathrm{OH}^{+}$ or ions from other residual gas molecules are observed. It is known that protonated water is the dominant cluster species emitted from pure water ice under ion and electron bombardment ${ }^{20}$ and also that on foreign substrates water clustering occurs at coverages less than $10 \%$ of a monolayer. ${ }^{21}$ 
Therefore, the ion spectra observed indicate the special role played by water molecules adsorbed on the surface in the decay properties of the van der Waals condensed gas films. It is not the intention of this paper to evaluate the very rich behavior of the protonated water clusters as a function of the various parameters (temperature, time, etc.), but a few comments are in order.

Whereas water cluster ions have been observed even at pressures in the low $10^{-9}$ mbar range and at all but the highest investigated moderator temperatures, ions of moderator atoms or molecules, which are orders of magnitude more abundant, could be detected only under special conditions. For instance no $\mathrm{Xe}$ or $\mathrm{Kr}$ ions emitted from the corresponding moderator have been observed. In the case of $\mathrm{Ar}$ and $\mathrm{N}_{2}$ only a single weak peak corresponding to $\mathrm{Ar}^{+}$and $\mathrm{N}_{2}^{+}$, respectively, is visible and this only if a fresh layer deposited near the sublimation temperature is used. The weak intensity is due to the fact that, in contrast to the case of water ionization, for RGS and $\mathrm{N}_{2}$ single ion excitations are strongly quenched and the less probable multiple excitation processes (such as multiple ionization, simultaneous ionization and excitation, creation of exciton pairs) are necessary to produce charged ion desorption of these species. ${ }^{22,23}$ The observed temperature and time dependence reflects the dynamical evolution of the moderator surface with these parameters and indicates that a detailed study of the ion spectra as a function of various parameters could be used to gain insight into the surface dynamics of condensed van der Waals moderators in the presence of adsorbates.

The ions represent only a small fraction of the ejected moderator material. Neutrals are much more intense and determine the amount of erosion experienced by the film. After traversing the $\mathrm{Al}$ substrate, the muons have a wide energy distribution with a mean energy of $\sim 600 \mathrm{keV}$. They typically deposit a few $\mathrm{keV}$ in the condensed gas film where this energy may initiate a cascade leading to the sputtering of atoms or molecules. Since for $\mu^{+}$beams we expect a similar sputtering behavior as for proton beams, we scaled proton sputtering data to estimate the extent of erosion. ${ }^{24,25}$ We conclude that in our case for $\operatorname{Ar} \sim 0.4$ atoms $/ \mu^{+}$, for $\mathrm{N}_{2} \sim 0.1$ molecules $/ \mu^{+}$and for $\mathrm{Xe} \sim 0.02$ atoms $/ \mu^{+}$are ejected from the condensed gas layer. These numbers are too low to produce significant erosion of the solidified gas layer. Another possible effect such as beam induced evaporation as a consequence of the heating of the moderator can be completely neglected in our case, due to the very low incident flux $\left(\sim 10^{6}-10^{7} \mu^{+} / \mathrm{s} \mathrm{cm}^{2}\right)$. That the decay properties are solely determined by the age of the layer has also been confirmed experimentally by switching off the beam and observing the time decay. No deviation from the variation in time with beam could be observed.

A versatile use of the very slow $\mu^{+}$as magnetic microprobes of thin film samples requires that the moderator remains stable also in an adverse environment. A frequent case is one of a hot sample. This could be tested during a measurement of the magnetization of thin Ni films as a function of temperature, where without special precautions a sample temperature as high as $700 \mathrm{~K}$ did not affect the cryogenic moderator in our apparatus.

\section{CONCLUSIONS}

Our investigations have yielded new and useful results on the moderation of polarized muons in condensed gas solids. A large value of the escape depth of epithermal muons from solid Ar and solid $\mathrm{Kr}$ films was found. Such large escape depths have been previously reported for slow positrons and $\mathrm{x}$-ray induced secondary electrons emitted from rare gas solids. ${ }^{5,26}$ Although the interaction mechanisms responsible for the slowing down and escape are different, the peculiar properties of rare-gas solids (wide band gap, weak van der Waals bonding, closed atomic shell) lead to favorable conditions for so many different particles. In this respect it would be interesting to perform similar detailed investigations on the moderation of protons in rare-gas solids. ${ }^{27}$ Protons with some $\mathrm{keV}$ kinetic energies are more readily available than muons and we expect that the understanding of the physics underlying the muon moderation and the related applications would benefit from such investigations.

For solid Ar and solid $\mathrm{Kr}$ we determined optimal growth conditions as a function of temperature. Furthermore, the moderation efficiencies were found to increase when the films were annealed after being deposited at $\sim 10 \mathrm{~K}$. While it was possible to observe a decay in time of the yield due to film contamination under nonoptimum UHV conditions, no time dependence was observed from radiation damage and sputtering. The decay can be completely suppressed by insuring UHV conditions during the operation. These results show that optimal stable operating conditions that are necessary for reliable operation and use of a very slow muon beam can be achieved with a reasonable technological effort. For instance the optimal temperature for $\mathrm{Ar}$ or $\mathrm{N}_{2}$ moderators can be obtained with a simple two-stage closed cycle cryostat and the UHV conditions required are standard for surface and thin film science.

\section{ACKNOWLEDGMENTS}

We thank F. Kottmann and U. Zimmermann for their help during the preliminary stages of this work. One of us (T.P.) acknowledges financial support by the Human Capital and Mobility Program (European Union) by the Swiss Federal Office of Education and Science and by the Land BadenWürttemberg. A.H., M.B., C.N., J.L. and G.S. acknowledge financial support by the Bundesministerium für Bildung und Forschung.

${ }^{1}$ D. R. Harshman, A. P. Mills, J. L. Beveridge, K. R. Kendall, G. D. Morris, M. Senba, J. B. Warren, A. S. Rupaal, and J. H. Turner, Phys. Rev. B 36, 8850 (1987).

${ }^{2}$ E. Morenzoni, F. Kottmann, D. Maden, B. Matthias, M. Meyberg, Th. Prokscha, Th. Wutzke, and U. Zimmerman, Phys. Rev. Lett. 72, 2793 (1994).

${ }^{3}$ E. Morenzoni, M. Birke, A. Hofer, F. Kottmann, J. Litterst, B. Matthias, M. Meyberg, C. Niedermayer, Th. Prokscha, G. Schatz, and Th. Wutzke, Hyperfine Interact. 97/98, 395 (1996).

${ }^{4}$ A. Schenck, Muon Spin Rotation Spectroscopy (Adam Hilger, Bristol, 1985).

${ }^{5}$ E.M. Gullikson and A. P. Mills, Jr., Phys. Rev. Lett. 57, 376 (1986).

${ }^{6}$ K. G. Lynn and B. Nielsen, Phys. Rev. Lett. 58, 81 (1987).

${ }^{7}$ G. Baldini, Phys. Rev. 137, A508 (1965).

${ }^{8}$ J.F. Ziegler, J.P. Biersack, and U. Littmark, The Stopping and Range of Ions in Solids (Pergamon, New York, 1985), Vol. 1. 
${ }^{9}$ Rare Gas Solids, edited by M. L. Klein and J. A. Venables (Academic, New York, 1977), Vol. II.

${ }^{10}$ I. Ya. Fugol, Adv. Phys. 27, 1 (1978).

${ }^{11}$ A. E. Cuzon and A. T. Pawlowicz, Proc. Phys. Soc. London 85, 375 (1965).

${ }^{12}$ S. I. Kovalenko and N. N. Bagrov, Sov. Phys. Solid State 11, 2207 (1970).

${ }^{13}$ W. Schulze and D. M. Kolb, Faraday Trans. II: Chem. Phys. 70, 1098 (1974).

${ }^{14}$ J. Hengevoss, J. Vac. Sci. Technol. 6, 58 (1969).

${ }^{15}$ K. Becker, G. Kippling, W. D. Schoenherr, W. Schulze, and V. Toelle, in Proceedings of the IVth International Cryogenic Engineering Conference, Eindoven, 1972, p. 319.

${ }^{16}$ A. P. Mills, Jr. , and E. M. Gullikson, Appl. Phys. Lett. 49, 1121 (1986).

${ }^{17}$ A. P. Mills, Jr., S. S. Voris, and T. S. Andrew, J. Appl. Phys. 76, 2556 (1994).

${ }^{18}$ J. Jääskeläinen, T. Laine, K. Fallström, K. Saarinen, and P. Hautojärvi, in
SLOPOS 7, Slow Positron Workshop, Unterägeri, Switzerland, 1996, Book of Abstracts.

${ }^{19}$ M. P. Petkov, K. G. Lynn, and L. O. Roellig, in SLOPOS 7, Slow Positron Workshop, Unterägeri, Switzerland, 1996, Book of Abstracts.

${ }^{20}$ G. M. Lancaster, F. Honda, Y. Fukuda, and J. W. Rabelais, Int. J. Mass Spectrophotom. Ion Phys. 29, 199 (1979).

${ }^{21}$ A. D. Bass and L. Sanche, J. Chem. Phys. 95, 2910 (1991).

${ }^{22}$ G. Dujardin, L. Hellner, M. J. Besnard-Ramage, and R. Azria, Phys. Rev. Lett. 64, 1289 (1990).

${ }^{23}$ R. A. Rosenberg, V. Rehn, A. K. Green, and P. R. LaRoe, Phys. Rev. Lett. 51, 915 (1983).

${ }^{24}$ J. Schou, Nucl. Instrum. Methods 27, 188 (1987).

${ }^{25}$ W. L. Brown and R. E. Johnson, Nucl. Instrum. Methods 13, 295 (1986).

${ }^{26}$ E. M. Gullikson and B. L. Henke, Phys. Rev. B 39, 1 (1989).

${ }^{27}$ A. P. Mills, Jr., M. Leventhal, M. Y. Lanzerotti, D. M. Zuckerman, E. M. Gullikson, and G. R. Brandes, Phys. Rev. B 42, 5973 (1990). 